\title{
Dynamic Texturing of Real Objects in an Augmented Reality System
}

\author{
Krešimir Matković \\ VRVis Research Center \\ Vienna, Austria \\ Matkovic@VRVis.at
}

\author{
Thomas Psik, Ina Wagner \\ Vienna University of Technology \\ Vienna, Austria \\ \{tpsik, iwagner\}@pop.tuwien.ac.at
}

\author{
Denis Gračanin \\ Virginia Tech \\ Blacksburg, VA 24061, USA \\ gracanin@vt.edu
}

\begin{abstract}
The ability to physically change properties of real objects used in augmented reality $(A R)$ applications is limited. Geometrical properties (shape, size) and appearance (color, texture) of a real object remain unchanged during a single application run. However, an AR system can be used to provide a virtual texture for the real object. The texture can be changed dynamically based on user interactions. The developed AR system includes two components, the "3D Table" and the "Texture Painter." The 3D Table is a table where real objects are placed. The tabletop is used as a projection surface, making it possible to add a context to the real object. The Texture Painter makes it possible to paint on the real object, using a real brush and virtual ink (texture). ARToolkit markers are placed on the $3 D$ Table tabletop to augment the environment with the virtual objects. Markers are either physical (printouts on the tabletop) or virtual (projections). The scene is recorded with a camera and the composed video is projected in real time. The projection shows a virtual environment, real objects painted with virtual ink, and virtual objects positioned where real or virtual ARToolkit markers are placed. The developed system is used in architectural design applications where, due to the different qualities of real architectural models and rendered architectural models, real models are still used. The system was tested at the Academy of Fine Arts in Vienna where it is used as a support tool for architecture students.
\end{abstract}

\section{Introduction}

Virtual Reality (VR) allows a user to immerse in and experience a completely synthetic virtual environment. However, the created virtual environment is still "simpler" than the real world. Unlike VR, Augmented Reality (AR) enables the user to interact with virtual objects and real objects in a real environment in real time [1]. The user can experience enhanced reality by adding virtual objects and by superimposing the computer generated information such as text or graphics onto real objects. A typical AR system combines several different components and technologies into a single system. That includes, for example, display technologies enabling the combination of real and virtual objects into a single view and a tracking system allowing real-time interaction, modeling and calibration.

One of the main problems with using real objects in an AR system is time and effort needed to change their properties. For example, one can not simply change the shape or size of a real object. However, some other properties like color and texture can be changed "virtually" so that all changes made in the virtual world would be automatically transferred to the real world, and vice versa. Dynamic coloring and texturing of real objects in AR systems open some interesting possibilities for interactive design and modeling, especially for applications where the size and shape of objects is relatively stable, i.e. it does not change during a single application run.

Architectural modeling and design is a typical example of such applications [3,7]. Architectural models (real objects) used to be very important in architects practice. They are being gradually replaced with the computer generated and rendered models but the models are still indispensable in some situations. Architects may prefer to keep real objects and real models instead of depending only on computers during the design process. They continuously transform the environment during the design process to experiment and create many unusual and unconventional combinations. This make architectural design an excellent application domain for the developed system developed together with architects and architecture students from the Academy of Fine Arts in Vienna.

\section{System Design}

Two main problems were addressed. First, a well defined physical environment must be provided for placing and manipulating real objects so that they can be integrated in an AR environment. Second, an "augmented brush" must be 
provided for interactive, dynamic "painting" of the real objects using colors, textures or animations/video clips. Consequently, the two main components of the developed system are the 3D Table and the Texture Painter. The Texture Painter can be used stand alone or in combination with the 3D Table (Figure 1).

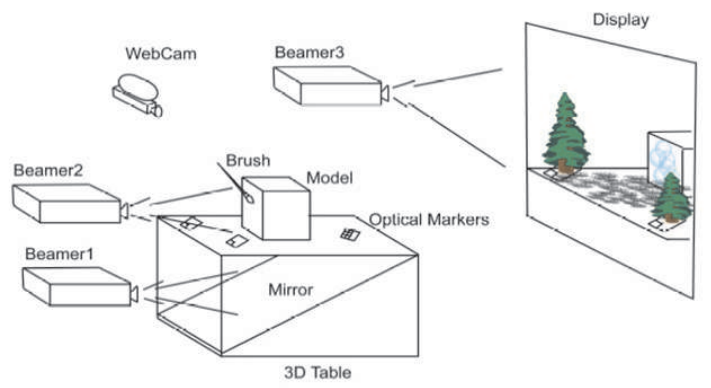

Figure 1. System design

\subsection{D Table}

The 3D Table is a central component of the system [6]. It is a table with a semi transparent glass top. The tabletop serves as a back-projection surface. The main idea is to use it as a modeling table. The real objects are painted using the Texture Painter and then the corresponding environment is created by projecting images on the tabletop. Any image can be used, for example city maps, various landscapes or abstract images. Animations and video clips are used to show the models in dynamic context. The system is implemented so that the model can be illuminated only from one side, the side visible to a user. A mirror inside the table makes it possible to use a beamer (video projector) placed next to the table to project images on the tabletop. The USB connectors are used to connect cameras. ARToolKit [4] physical markers can be placed on the tabletop.

\subsection{Texture Painter}

Texture Painter is an application used to paint virtual ink on real objects using a real brush. A user has a real brush in the hand, and "paints" real objects with it. Instead of using a real paint, textures (static images or videos) are applied. The installation includes a projector, a brush and an object that will be painted. The brush is a slightly modified conventional brush. A retro reflective marker has to be put on the brush to make it possible to precisely track it. A simple camera was used to track the brush in the first implementation. The current implementation uses DynaSight ${ }^{T M}$ sensor from Origin Instruments with which the brush tracking works very stable and smoothly. Since point and click is needed for painting, just as in the most interfaces based on the common WIMP paradigm, an additional button is needed. During initial experiments a wireless mouse was used as a click device (only the buttons were used). The system worked well, but it was inconvenient to hold the mouse in the hand all the time. The next step was adding a button and a radio transmitter to the brush.

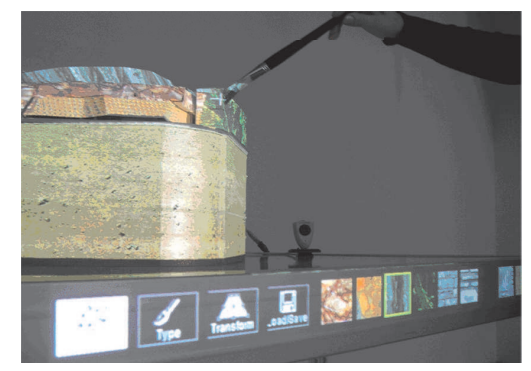

Figure 2. Using a texture brush

Figure 2 shows an example of using a texture brush. Before a design process can start, a projector and the real object (architectural model) have to be positioned. The brush tracking device is placed on the top of the projector and the system needs to be calibrated.

The perspective distortions occurring at the real object surfaces which are not perpendicular to the projection axis are not taken into account. It would be possible to track the real object and use object geometry information[6] but the emphasis is on systems simplicity so it can be easily used by non-expert users.

Figure 2 also shows a system toolbar projected on the table side. The toolbar displays available textures and tools. After the user has selected a texture (using the brush and the button), the object will be painted at the position of the brush, when the button is pressed. Additional functionality such as polygon draw, polygon fill, and other well known functionality from basic painting programs were implemented as well. This speeds up the paint process in case of large planes that need to be covered with the same texture.

\subsection{Markers}

The arranged scene consists of the painted object, the background projected on the surface of the table and the physical markers with the over imposed virtual objects. Since the 3D Table is capable of back projection, not only physical markers but the virtual (projected) markers can be used. The physical markers have the advantage that they can be manipulated in a tangible way, just by moving them on the table. However, a scene can only be restored by replacing all physical markers as they were before. The advantage of virtual markers is that the current marker configuration can be saved and reloaded later. That enables a "version 
control" where various stages in design evolution and development can be saved and reloaded at will. The version control provides an insight into a design process and helps in educational process.

The scene can be explored using one or more cameras. For each camera a video stream displaying the whole scene, including context image, painted object, and virtual 3D objects superimposed on the markers, is rendered. A snapshot and video export functions are implemented to document the current state.

\section{User Interface}

The Texture Painter can be made more advanced [1, 4], but the aim was to make a simple, low-cost solution, which can be setup anywhere straightforward by non-expert users. Furthermore, the object geometry is not needed which speeds up the process and allows rapid and straightforward experiments. Interestingly, architects didnt complain about the drawbacks, they use the tool extensively. Simplicity and mobility of the setup makes it popular among the students.

As there is no real object geometry stored in the system, object polygons can not be automatically found. The user has to specify polygon vertexes using the brush, and this polygon will be filled. If the user wants to scale or rotate the texture, the user selects the rotation or scale tool from the toolbar. By moving the brush closer or further to the projector, the texture is scaled or rotated accordingly.

Texture manipulation plays an important role in the design process. Imagine, for example, a small white block painted with a brick texture. If the texture is scaled, so that the cube contains only 3 rows of bricks, it will be perceived as relatively small. If the texture is scaled down, the same block suddenly appears to be a wall. If the process goes on in either direction (making the bricks very small or huge) the block will be not be perceived as a block made of bricks any more. Playing in this way with individual textures and combinations of textures is common and important architectural practice.

The ability to combine and manipulate a real object and textures together is one of the most useful features of the system. Although texture selections using a mouse and a brush are different, the underlying metaphor is the same.

While most of the tangible AR interfaces require the user to wear a head-mounted display (HMD) in order to view the AR environment [2], the developed system overcomes this by using two different approaches. In the first approach, the AR environment is projected on the projection screen to provide a simple visual feedback. Similar approach is used in the AR Groove application [5]. However, using a separate projection screen removed from the working environment may make it difficult for the user to observe results of interactions with the system. The second approach uses the 3D Table projector to directly project textures on the real object (tangible interface) and eliminates any need for an HMD or a projection screen. There is no separation between the display and the working environment. Interactions are intuitive and the user can directly observe results.

The user interface provides for direct manipulation and alleviates some of the related concerns [8]. The relative simplicity of the system means that the required system resources are relatively smaller compared to similar systems. Since a user uses a real brush and interacts with the system as if painting objects surfaces, users actions are natural and intuitive. Save and load features for virtual markers enable, to some extent, history and tracing mechanisms. Texture combination and scaling with a real object provide useful design macros. Direct texture projection onto the real object makes the system more accessible to users with limited eyesight (no need to use an HMD or a projection screen).

\section{Case Study}

The system was used by approximately 20 architecture students from the Academy of Fine Arts in Vienna. The overall simplicity was imperative. The whole system is very simple and any non-expert user can start using it immediately. Students can bring architectural models, just place them on the tabletop, and start painting. There is no need for additional trackers or 3D scanning.

The whole setup, as illustrated in Figure 1, consists of the 3D Table where a projector projects a landscape onto the tabletop. A physical architectural model is placed on the table. The projected video contains the painted architectural model, the landscape, and the virtual objects in the places where real or virtual markers are. Virtual markers simulate different scales easily, and they can be used to restore physical markers positions after save and load.

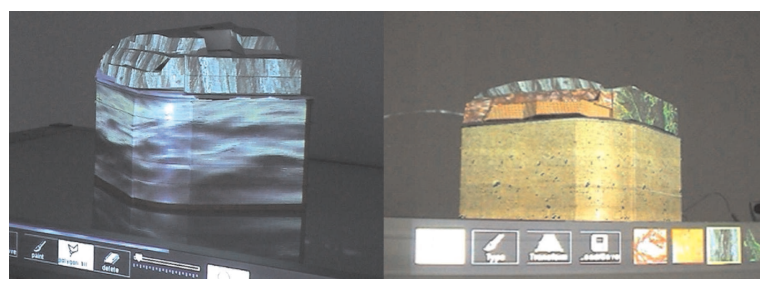

\section{Figure 3. Different textures significantly changing the same model}

Figure 3 shows examples of different textures applied to an architectural model. The underlying model is always the same, but due to the different textures the overall impression is quite different. Doing these experiments using only a traditional model and real textures would be much more tedious. The model used is an existing design for which 
the architects originally explored design issues by producing and studying sketches and collages. The design process was re-enacted using the developed system. That included accentuating a difference between the base and the attachment as well as studying the possibilities to merge different parts of the design. The design process included painting a variety of different textures onto the base and different parts of the attachments while exploring their changing relationship. The projections of different textures charged the building with different meanings [7].

Central design issues were choice of materials (appearance) and the duality of base (the building) and attachments (the attic). Texturing a base of the model using a video texture showing waves transformed the model into a cliff with a fortress or a concrete structure on the top of the cliff. Changing the context also changes the scale, from building to cliff. The projections helped erase preconceptions of the building, seeing it differently.

In other studies some of the issues included how to make the complex interior 3D structure of apartments, patios and terraces visible outside. Other concerns were the materiality of the faade and the possibilities of differences through using textures. While the system is very simple and easy to use, case studies pointed to some problems. The disadvantage is that textures do not follow the architectural model when it is moved. The textures are also sometimes distorted in terms of perspective. The experiments with students show that these disadvantages are not significant and that all of the students are very fond of using the system.

\section{Conclusions and Future Work}

This paper describes an AR system built to support architectural design process. The real object, an architectural model with its own qualities, still plays an important role in this process. Computer generated renderings simply cannot replace the architectural model in all cases. Instead of replacing the architectural model, the AR system is used to enhance it. Actually, the enhancement goes in both directions. Not only the architectural models are enriched, but the computer generated images projected on the architectural model (instead on the plain projection screen) are much more vivid and useful. This system makes an inspiring test bed for the architects. The architectural models can be significantly enhanced by using the Texture Painter. The ease of painting and the natural user interface makes it a very popular tool among the students. This demonstrates how an interesting combination of simple tools can be used to create an innovative AR system, and to reach a new and broader group of users. Although none of the components are novel in the field, the unique combination, low cost and the demonstration of the ease of use is the main contribution of the described system.
The future work will involve studying connections and relationships among different parts of the system. If a user changes the scale of a texture on the real object, the system should be able to automatically change the size of the virtual markers. Another interesting field of exploration is "saving" the physical markers. After "telling" the system that a scene should be saved, all physical markers can be removed and virtual markers will be added to the background at the positions extracted from the physical markers.

\section{Acknowledgments}

The authors would like to thank our co-researchers from the Atelier Project, in particular Andreas Rumpfhuber. This work was partially sponsored by the European Commission - IST programme - FET - Proactive Initiative - The Disappearing Computer II - through the ATELIER project (EU IST-2001-33064). Parts of this work were carried out at the VRVis Research Center in Vienna, Austria, which is funded by an Austrian governmental research program called $\mathrm{K}$ plus. This work was also partially sponsored by the National Institutes of Health (NIH) grant 5R03LM008140-02.

\section{References}

[1] M.-O. Berger, B. Wrobel-Dautcourt, S. Petitjean, and G. Simon. Mixing synthetic and video images of an outdoor urban environment. Machine Vision Applications, 11(3):145-159, 1999.

[2] D. A. Bowman, E. Kruijff, J. J. LaViola, Jr., and I. Poupyrev. $3 D$ User Interfaces: Theory and Practice. Addison-Wesley, Boston, 2004.

[3] G. Iacucci and I. Wagner. Supporting collaboration ubiquitously: an augmented learning environment for architecture students. In Proceedings of the Eight European Conference on Computer Supported Cooperative Work (ECSCW) 2003, pages 139-159, 2003.

[4] H. Kato, M. Billinghurst, I. Poupyrev, K. Imamoto, and K. Tachibana. Virtual object manipulation on a table-top ar environment. In Proceedings of ISAR 2000, 2000.

[5] I. R. Poupyrev, R. Berry, J. Kurumisawa, K. Nakao, M. Billinghurst, C. Airola, H. Kato, T. Yonezawa, and L. Baldwin. Augmented groove: Collaborative jamming in augmented reality. In SIGGRAPH 2000 Conference Abstract and Applications, page 77. ACM Press, 2000.

[6] R. Raskar, G. Welch, and W. Chen. Table-top spatially augmented reality: bringing physical models to life with projected imagery. In Proceedings of Second IEEE and ACM International Workshop on Augmented Reality (IWAR99), 1999.

[7] A. Rumpfhuber and I. Wagner. Sampling mixed objects as part of architectural practice. In Proceedings of Pixelreiders 2004, 2004.

[8] B. Schneirdeman. Designing the User Interface: Strategies for Effective Human-Computer Interaction. Addison-Wesley, Reading, Massachusetts, third edition, 1998. 